\title{
Classification of bijections between 321- and 132-avoiding permutations ${ }^{\dagger}$
}

\author{
Anders Claesson and Sergey Kitaev \\ The Mathematics Institute, Reykjavik University, Kringlan 1, 103 Reykjavik, Iceland
}

\begin{abstract}
It is well-known, and was first established by Knuth in 1969, that the number of 321-avoiding permutations is equal to that of 132 -avoiding permutations. In the literature one can find many subsequent bijective proofs confirming this fact. It turns out that some of the published bijections can easily be obtained from others. In this paper we describe all bijections we were able to find in the literature and we show how they are related to each other (via "trivial" bijections). Thus, we give a comprehensive survey and a systematic analysis of these bijections. We also analyze how many permutation statistics (from a fixed, but large, set of statistics) each of the known bijections preserves, obtaining substantial extensions of known results.

We also give a recursive description of the algorithmic bijection given by Richards in 1988 (combined with a bijection by Knuth from 1969). This bijection is equivalent to the celebrated bijection of Simion and Schmidt (1985), as well as to the bijection given by Krattenthaler in 2001, and it respects 11 statistics (the largest number of statistics any of the bijections respect).
\end{abstract}

Keywords: bijection, permutation statistics, equidistribution, pattern avoidance, Catalan structures

\section{Introduction}

Given two different bijections between two sets of combinatorial objects, what does it mean to say that one bijection is better than the other? Perhaps, a reasonable answer would be "The one that is easier to describe." While the ease of description and how easy it is to prove properties of the bijection using the description is one aspect to consider, an even more important aspect, in our opinion, is how well the bijection reflects and translates properties of elements of the respective sets.

A natural measure for a bijection between two sets of permutations, then, is how many statistics the bijection preserves. Obviously, we don't have an exhaustive list of permutation statistics, but we have used the following list as our "base" set:

$$
\begin{aligned}
& \text { asc, des, exc, ldr, rdr, lir, rir, zeil, comp, lmax, lmin, rmax, rmin, } \\
& \text { head, last, peak, valley, lds, lis, rank, cyc, fp, slmax . }
\end{aligned}
$$

\footnotetext{
${ }^{\dagger}$ The research presented here was supported by grant no. 060005012 from the Icelandic Research Fund 1365-8050 (c) 2008 Discrete Mathematics and Theoretical Computer Science (DMTCS), Nancy, France
} 
These are defined in Section 2. To make sure we find all statistics that a given bijection "essentially" preserves, we include in our list of statistics those that are obtained from our "basic" statistics by applying to them the trivial bijections on permutations (reverse $=r$, complement $=c$, inverse $=i$ ) and their compositions. Moreover, for each statistic, stat, in this extended list we consider two other statistics: $\mathrm{n}$-stat $(\pi)=n-\operatorname{stat}(\pi)$ and $\mathrm{m}-\operatorname{stat}(\pi)=n+1-\operatorname{stat}(\pi)$, where $n$ is the length of the permutation. The meaning of n-stat or m-stat is often "non-stat"; for example, $n$-fp counts non-fixedpoints.

This way each basic statistic gives rise to 24 statistics. The base set contains 23 statistics, giving a total of 552 statistics. There are, however, many statistics in that set that are equal as functions; for instance, des $=$ asc.r, and peak = peak.r = valley.c (we use a dot to denote composition of functions). Choosing one representative from each of the classes of equal statistics results in a final set of 190 statistics; we call this set STAT. In practice we settled for "empirical equality" when putting together STAT: we considered two statistics equal if they gave the same value on all 5914 permutations of length at most 7 .

In the theorems below, the statistics presented are linearly independent, and the results are maximal in that they cannot be non-trivially extended using statistics from STAT. That is, adding one more pair of equidistributed statistics from STAT to any of the results would create a linear dependency among the statistics.

A permutation $\pi=a_{1} a_{2} \ldots a_{n}$ avoids the pattern 321 if there are no indices $i<j<k$ such that $a_{k}<a_{j}<a_{i}$. It avoids 132 if there are no indices $i<j<k$ such that $a_{i}<a_{k}<a_{j}$. Avoidance of other patterns is defined similarly.

Knuth $(6 ; 7)$ showed that the number of permutations avoiding a pattern of length 3 is independent of the pattern. This number is the $n$-th Catalan number, $C_{n}=\frac{1}{n+1}\left(\begin{array}{c}2 n \\ n\end{array}\right)$. To prove this it suffices, due to symmetry, to consider one representative from $\{123,321\}$ and one from $\{132,231,213,312\}$. The symmetry also means that to prove this bijectively, it suffices to find a bijection from the set of permutations avoiding a pattern in one of the classes to permutations avoiding a pattern in the other. This turns out to be a rather complicated problem. Several authors have, however, succeeded in doing so $(4 ; 5 ; 8 ; 10 ; 11 ; 13 ; 14 ; 15)$; we describe all those bijections in Section 3. The following result shows that many of these bijections are "trivially" related:

Theorem 1 The following relations among bijections between 321-and 132-avoiding permutations hold:

$$
\begin{aligned}
\text { reverse } \circ \Phi^{-1} & =\text { inverse } \circ \text { Simion-Schmidt } \circ \text { reverse } \\
& =\text { inverse } \circ \text { Krattenthaler } \circ \text { reverse } \circ \text { inverse } \\
& =\text { inverse } \circ \text { reverse } \circ \text { Mansour-Deng-Du } \\
& =\text { Knuth-Richards }{ }^{-1} \circ \text { reverse }
\end{aligned}
$$

and

$$
\text { Reifegerste }=\text { inverse } \circ \text { Knuth-Rotem } \circ \text { inverse } .
$$

In the theorem below we regard all bijections as bijections from 321- to 132-avoiding permutations; we apply trivial transformations if a bijection has a different domain or codomain, see Theorem 1 above.

Theorem 2 For bijections from 321- to 132-avoiding permutations we have the following equidistribution results. These results are maximal in the sense that they cannot be non-trivially extended by other statistics from STAT. 
(11) $\Phi$, Knuth-Richards, Krattenthaler, Mansour-Deng-Du, Simion-Schmidt

peak.i valley rank.r comp lir.i rir last exc slmax.r.c slmax.i head.i

valley valley.i rank $\operatorname{rmax} \operatorname{ldr} \operatorname{ldr} . \mathrm{i}$ head.i asc lir.i lir $\operatorname{lmax}$

\section{(9) Knuth-Rotem, Reifegerste}

valley peak.i exc slmax.i head.i slmax.r.c rir lir.i last valley valley.i des zeil ldr $\quad \mathrm{dr} \quad \operatorname{rmin} \operatorname{lmax}$ m-ldr.i

(7) West

peak.i exc slmax.i slmax.r.c rir lir.i last

valley.i asc lir.i comp rmax ldr.i head

(5) Knuth

exc fp lir.i lir lis

exc fp rmin lmax n-rank

(1) Elizalde-Deutsch

fp

fp

In Section 2 we define the relevant statistics; in Section 3 we describe the bijections; and in Section 4 we give a new recursive description of the bijection by Knuth and Richards.

\section{Permutation statistics}

Let us define:

asc $=$ number of ascents (elements followed by a larger element);

comp $=$ number of components (ways of factoring $\pi=\sigma \tau$ so that each letter in $\sigma \neq \epsilon$ is smaller than each letter in $\tau$ );

des $=$ number of descents (elements followed by a smaller element);

exc $=$ number of excedances (positions $i$ in $\pi$ such that $\pi(i)>i$ );

$\mathrm{fp}=$ number of fixed points (positions $i$ in $\pi$ such that $\pi(i)=i$ );

head $=$ first (leftmost) element;

last $=$ last (rightmost) element;

ldr $=$ length of the leftmost decreasing run (largest $i$ such that $\pi(1)>\pi(2)>\cdots>\pi(i))$;

lds = length of the longest decreasing sequence in a permutation;

lir $=$ length of the leftmost increasing run (largest $i$ such that $\pi(1)<\pi(2)<\cdots<\pi(i)$ );

lis $=$ length of the longest increasing sequence in a permutation;

$\operatorname{lmax}=$ number of left-to-right maxima (number of indices $j$ in $\{1, \ldots, n\}$ such that $\pi(i)<\pi(j)$ whenever $1 \leq i<j$ ); 


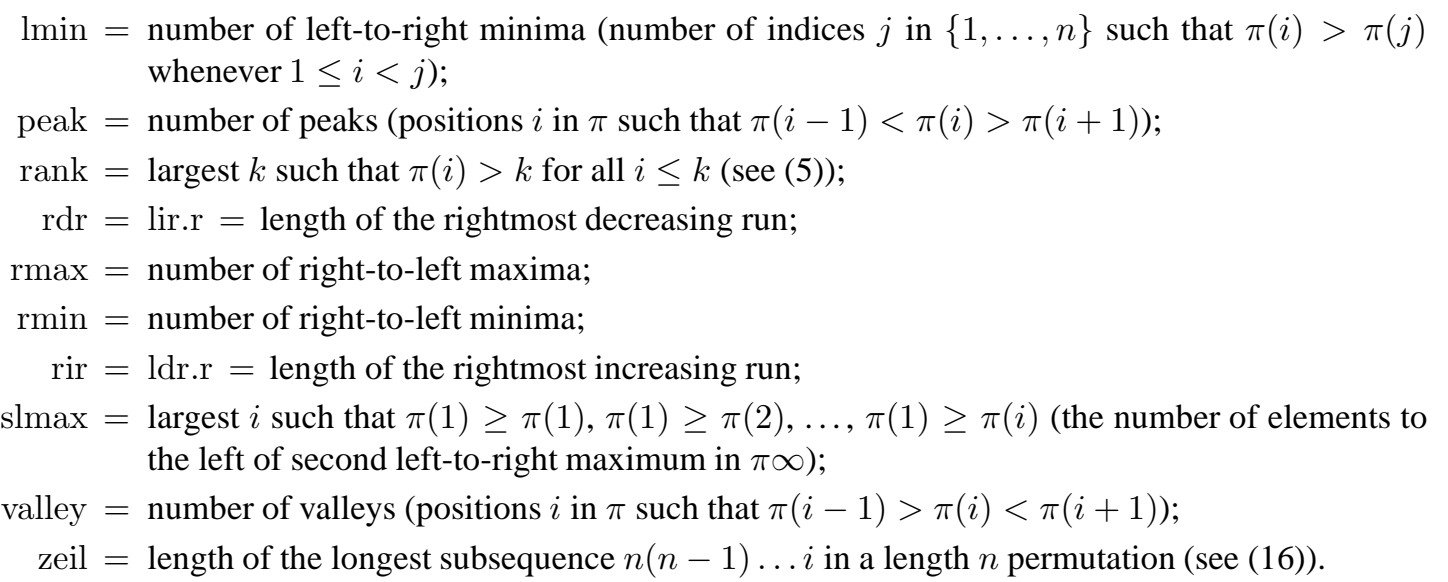

Let us also describe some of the derived statistics:

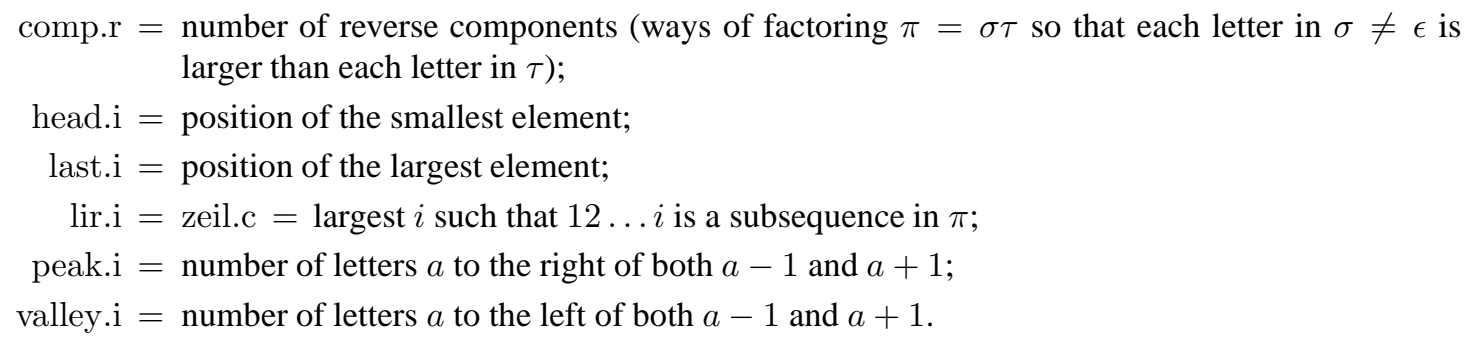

\section{Bijections in the literature}

In this section we describe the bijections, and we try to stay close to the original sources when doing so. In what follows $\mathcal{S}_{n}(\tau)$ is the set of $\tau$-avoiding permutations of length $n$, and $\mathcal{D}_{n}$ is the set of Dyck paths of length $2 n$.

\subsection{Knuth's bijection, 1973}

Knuth (6, pp. 242-243) gives a bijection from 312-avoiding permutations to "stack words". Formulated a bit differently, it amounts to a bijection from 132-avoiding permutations to Dyck paths. Knuth (7, pp. 60-61) also gives a bijection from 321-avoiding permutations to Dyck paths. By letting permutations that are mapped to the same Dyck path correspond to each other a bijection between 321- and 132-avoiding permutation is obtained-we call it Knuth's bijection.

We start by describing the bijection from 132-avoiding permutations to Dyck paths. We shall refer to it as the standard bijection. (This bijection is the same as the one given by Krattenthaler (8), who, however, gives a non-recursive description of it; see Section 3.6.) Let $\pi=\pi_{L} n \pi_{R}$ be a 132-avoiding permutation of length $n$. Each element of $\pi_{L}$ is larger than any element of $\pi_{R}$, or else a 132 pattern would be formed. We define the standard bijection $f$ recursively by $f(\pi)=u f\left(\pi_{L}\right) d f\left(\pi_{R}\right)$ and $f(\epsilon)=\epsilon$. Here, and elsewhere, $\epsilon$ denotes the empty word/permutation. Thus, under the standard bijection, the position of 
the largest element in a 132-avoiding permutation determines the first return to $x$-axis and vice versa. For instance,

$$
\begin{array}{r}
f(7564213)=u d f(564213)=u d u f(5) d f(4213)=u d u u d d u d f(213) \\
=u d u u d d u d u f(21) d=u d u u d d u d u u d u d d=
\end{array}
$$

As mentioned, Knuth also gives a bijection from 321-avoiding permutations to Dyck paths: Given a 321 -avoiding permutation, start by applying the Robinson-Schensted-Knuth correspondence to it. This classic correspondence gives a bijection between permutations $\pi$ of length $n$ and pairs $(P, Q)$ of standard Young tableaux of the same shape $\lambda \vdash n$. As is well known, the length of the longest decreasing subsequence in $\pi$ corresponds to the number of rows in $P$ (or $Q$ ). Thus, for 321-avoiding permutations, the tableaux $P$ and $Q$ have at most two rows.

The insertion tableau $P$ is obtained by reading $\pi=a_{1} a_{2} \ldots a_{n}$ from left to right and, at each step, inserting $a_{i}$ to the partial tableau obtained thus far. Assume that $a_{1}, a_{2}, \ldots, a_{i-1}$ have been inserted. If $a_{i}$ is larger than all the elements in the first row of the current tableau, place $a_{i}$ at the end of the first row. Otherwise, let $m$ be the leftmost element in the first row that is larger than $a_{i}$. Place $a_{i}$ in the square that is occupied by $m$, and place $m$ at the end of the second row. The recording tableau $Q$ has the same shape as $P$ and is obtained by placing $i$, for $i$ from 1 to $n$, in the position of the square that in the construction of $P$ was created at step $i$ (when $a_{i}$ was inserted). For example, the pair of tableaux corresponding to the 321-avoiding permutation 3156247 we get by the following sequence of insertions:

$$
\begin{aligned}
(\epsilon \mid \epsilon) \rightarrow(3 \mid 1) \rightarrow\left(\begin{array}{l|l}
1 & 1 \\
3 & 2
\end{array}\right) & \rightarrow\left(\begin{array}{l|l}
15 & 13 \\
3 & 2
\end{array}\right) \rightarrow\left(\begin{array}{l|l}
156 & 134 \\
3 & 2
\end{array}\right) \\
& \rightarrow\left(\begin{array}{l|l}
126 & 134 \\
35 & 25
\end{array}\right) \rightarrow\left(\begin{array}{l|l}
124 & 134 \\
356 & 256
\end{array}\right) \rightarrow\left(\begin{array}{l|l}
1247 & 1347 \\
356 & 256
\end{array}\right) .
\end{aligned}
$$

The pair of tableaux $(P, Q)$ is then turned into a Dyck path $D$. The first half, $A$, of the Dyck path we get by recording, for $i$ from 1 to $n$, an up-step if $i$ is in the first row of $P$, and a down-step if it is in the second row. Let $B$ be the word obtained from $Q$ in the same way but interchanging the roles of $u$ and $d$. Then $D=A B^{r}$ where $B^{r}$ is the reverse of $B$. Continuing with the example above we get

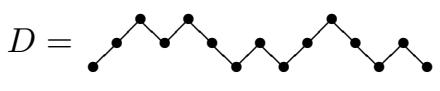

Elizalde and Pak (5) use this bijection together with a slight modification of the standard bijection to give a combinatorial proof of a generalization of the result by Robertson et al. (12) that fixed points have the same distribution on 123- and 132-avoiding permutations. The modification they use is to reflect the Dyck path obtained from the standard bijection with respect to the vertical line crossing the path in the middle. (Alternatively, the path can be read from the permutation diagram as described in (5).) We follow Elizalde and Pak and apply the same modification. After reflection, the path $f(7564213)$ above is the same as the path $D$ in the preceding example. Thus the image of the 321-avoiding permutation 3156247 under what we call Knuth's bijection is the 132-avoiding permutation 7564213 . 


\subsection{Knuth-Rotem's bijection, 1975}

Rotem (13) gives a bijection between 321-avoiding permutations and Dyck paths (described below). Combining it with the standard bijection gives a bijection from 321- to 132-avoiding permutations-we call it Knuth-Rotem's bijection.

A ballot-sequence $b_{1} b_{2} \ldots b_{n}$ satisfies $b_{1} \leq b_{2} \leq \cdots \leq b_{n}$ and $0 \leq b_{i} \leq i-1$, for $i=1,2, \ldots, n$. Let $\pi=p_{1} p_{2} \ldots p_{n}$ be a 321-avoiding permutation. From it we construct a ballot-sequences: Let $b_{1}=0$. For $i=2, \ldots, n$, let $b_{i}=b_{i-1}$ if $p_{i}$ is a left-to-right maximum in $\pi$, and let $b_{i}=p_{i}$ otherwise.

For the permutation $\pi=2513476$ we get the ballot-sequences 0013446 . This sequence we represent by a "bar-diagram", which in turn can be viewed as a lattice path from $(0,0)$ to $(7,7)$ :

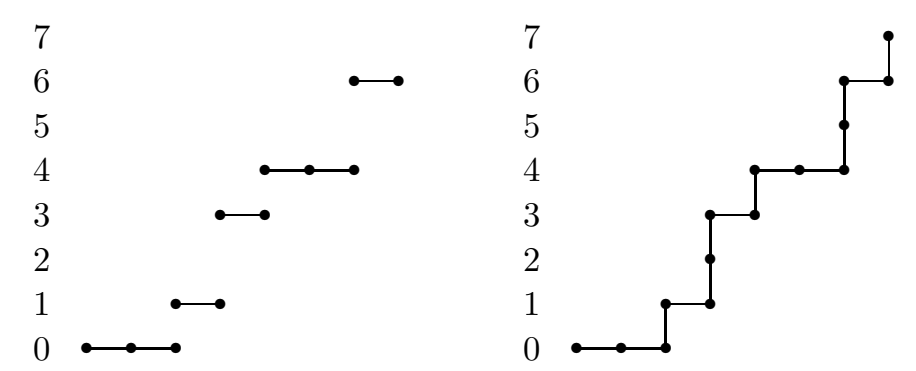

Rotating that path counter clockwise by $3 \pi / 4$ radians we get

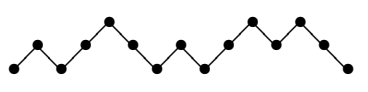

In the previous subsection we saw that this path is $f(7564213)$ where $f$ is the standard bijection. Thus the image of the 321-avoiding permutation 2513476 under Knuth-Rotem's bijection is the 132-avoiding permutation 7564213 .

\subsection{Simion-Schmidt's bijection, 1985}

Input: A permutation $\sigma=a_{1} a_{2} \ldots, a_{n}$ in $\mathcal{S}_{n}(123)$.

Output: A permutation $\tau=c_{1} c_{2} \ldots c_{n}$ in $\mathcal{S}_{n}(132)$.

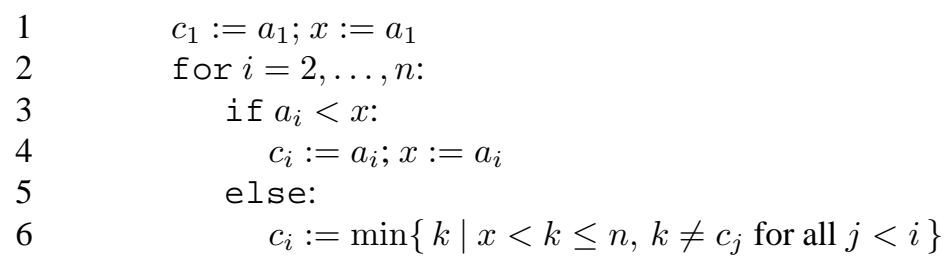

The map $\sigma \mapsto \tau$ defined by this algorithm is the Simion-Schmidt bijection (14). As an example, the 123 -avoiding permutation 6743152 maps to the 132 -avoiding permutation 6743125 .

\subsection{Knuth-Richards' bijection, 1988}

Richards' bijection (11) from Dyck paths to 123-avoiding permutations is given by this algorithm: 


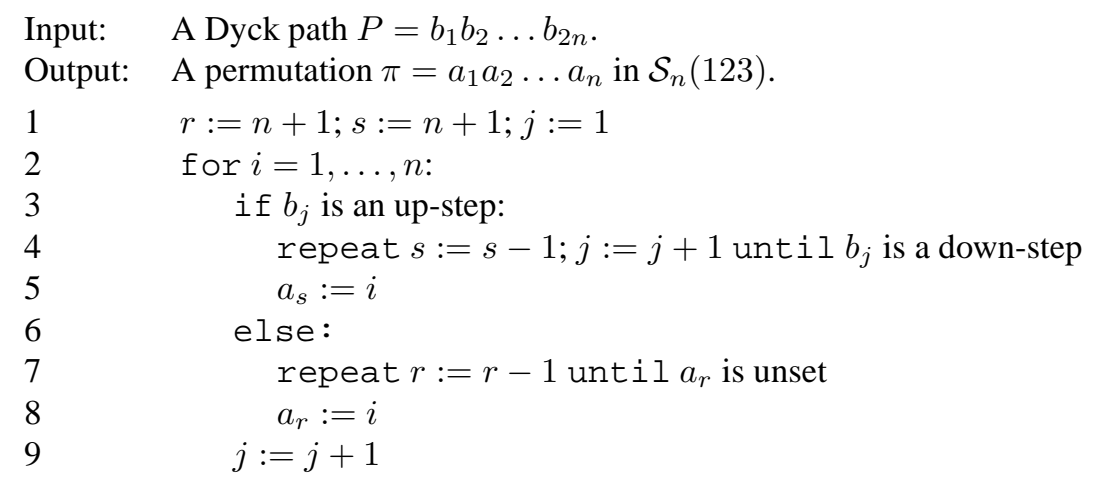

The Knuth-Richards bijection, from $\mathcal{S}_{n}(132)$ to $\mathcal{S}_{n}(123)$, is defined by Knuth-Richards = Richards $\circ f$, where $f$ is the standard bijection from 132-avoiding permutations to Dyck paths, and Richards is the algorithm just described. As an example, applying Knuth-Richards' bijection to 6743125 yields 5743612 .

\subsection{West's bijection, 1995}

West's bijection (15) is induced by an isomorphism between generating trees. The two isomorphic trees generate 123- and 132-avoiding permutations, respectively. We give a brief description of that bijection: Given a permutation $\pi=p_{1} p_{2} \ldots p_{n-1}$ and a positive integer $i \leq n$, let $\pi^{i}=p_{1} \ldots p_{i-1} n p_{i} \ldots p_{n-1}$; we call this inserting $n$ into site $i$. With respect to a fixed pattern $\tau$ we call site $i$ of $\pi$ in $\mathcal{S}_{n-1}(\tau)$ active if the insertion of $n$ into site $i$ creates a permutation in $\mathcal{S}_{n}(\tau)$.

For $i=0, \ldots, n-1$, let $a_{i+1}$ be the number of active sites in the permutation obtained from $\pi$ by removing the $i$ largest elements. The signature of $\pi$ is the word $a_{0} a_{1} \ldots a_{n-1}$. West (15) showed that for 123 -avoiding permutations, as well as for 132 -avoiding permutations, the signature determines the permutation uniquely. This induces a natural bijection between the two sets. For example, the 123avoiding permutation 536142 corresponds to the 132 -avoiding permutation 534612 -both have the same signature, 343322 .

\subsection{Krattenthaler's bijection, 2001}

Krattenthaler's bijection (8) uses Dyck paths as intermediate objects. Permutations that are mapped to the same Dyck path correspond to each other under this bijection.

The first part of Krattenthaler's bijection goes from 123-avoiding permutations to Dyck paths. Reading right to left, let the right-to-left maxima in $\pi$ be $m_{1}, m_{2}, \ldots, m_{s}$, so that $\pi=w_{s} m_{s} \ldots w_{2} m_{2} w_{1} m_{1}$, where $w_{i}$ is the subword of $\pi$ in between $m_{i+1}$ and $m_{i}$. Since $\pi$ is 123 -avoiding, the elements in $w_{i}$ are in decreasing order. Moreover, all elements of $w_{i}$ are smaller than those of $w_{i+1}$.

Read $\pi$ from right to left. Any right-to-left maximum $m_{i}$ is translated into $m_{i}-m_{i-1}$ up-steps (with $m_{0}=0$ ). Any subword $w_{i}$ is translated into $\left|w_{i}\right|+1$ down-steps, where $\left|w_{i}\right|$ denotes the number of elements of $w_{i}$. Finally, the resulting path is reflected in a vertical line through the center of the path.

The second part of Krattenthaler's bijection goes from 132-avoiding permutations to Dyck paths. Read $\pi=p_{1} p_{2} \ldots p_{n}$ in $\mathcal{S}_{n}(132)$ from left to right and generate a Dyck path. When $p_{j}$ is read, adjoin, to the path obtained thus far, as many up-steps as necessary to reach height $h_{j}+1$, followed by a down-step to height $h_{j}$ (measured from the $x$-axis); here $h_{j}$ is the number of elements in $p_{j+1} \ldots p_{n}$ which are 
larger than $p_{j}$. This procedure can be shown to be equivalent to the standard bijection from 132-avoiding permutations to Dyck paths.

For instance, Krattenthaler's bijection sends the permutation 536142 in $\mathcal{S}_{6}(123)$ to the permutaion 452316 in $\mathcal{S}_{6}(132)$ —both map to the same Dyck path, uuudduuddudd.

\subsection{Reifegerste's bijection, 2002}

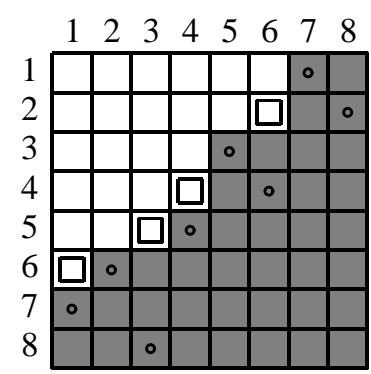

This figure illustrates Reifegerste's bijection (10). It pictures the 321avoiding permutation $\pi=13256847$ and the 132 -avoiding permutation $\pi^{\prime}=78564213$, two permutations that correspond to each other under that bijection.

Let $\pi=a_{1} a_{2} \ldots a_{n}$ be a 321 -avoiding permutation, and let $E$ be the set of pairs

$$
E=\left\{\left(i, a_{i}\right) \mid i \text { is an excedence }\right\} .
$$

For each pair $\left(i, a_{i}\right)$ in $E$, we place a square, called an $E$-square, in position $\left(i, n+1-a_{i}\right)$ in an $n \times n$ permutation matrix. ( $E$ uniquely determines $\pi$.) Next we shade each square $(a, b)$ of the matrix where there are no $E$-squares in the region $\{(i, j) \mid i \geq a, j \geq b\}$, thus obtaining a Ferrer's diagram. Finally, we get the 132-avoiding permutation $\pi^{\prime}$ corresponding to $\pi$ by placing dots (circles), row by row starting from the first row, in the leftmost available shaded square such that there are no two dots in any column or row. If $(i, j)$ contains a dot, then $\pi^{\prime}(i)=j$.

\subsection{Elizalde-Deutsch's bijection, 2003}

Here is an outline of a bijection by Elizalde and Deutsch (4): Map 321- and 132-avoiding permutation bijectively to Dyck paths; use an automorphism $\Psi$ on Dyck paths; and match permutations with equal paths.

We start by describing the automorphism $\Psi$. Let $P$ be a Dyck path of length $2 n$. Each up-step of $P$ has a corresponding down-step in the sense that the path between the up-step and the down-step form a proper Dyck path. Match such pairs of steps. Let $\sigma$ in $\mathcal{S}_{2 n}$ be the permutation defined by $\sigma_{i}=(i+1) / 2$ if $i$ is odd, and $\sigma_{i}=2 n+1-i / 2$ otherwise. For $i$ from 1 to $2 n$, consider the $\sigma_{i}$-th step of $P$. If the corresponding matching step has not yet been read, define the $i$-th step of $\Psi(P)$ to be an up-step, otherwise let it be a down-step. For example, $\Psi($ uuduudududddud $)=$ uuuddduduuddud.

The bijection $\psi$ from 321-avoiding permutations to $\mathcal{D}_{n}$ is defined as follows. Any permutation $\pi$ in $\mathcal{S}_{n}$ can be represented as an $n \times n$ array with crosses in the squares $(i, \pi(i))$. Given the array of $\pi$ in $\mathcal{S}_{n}(321)$, consider the path with down- and right-steps along the edges of the squares that goes from the upper-left corner to the lower-right corner of the array leaving all the crosses to the right and remaining always as close to the main diagonal as possible. Then the corresponding Dyck path is obtained from this path by reading an up-step every time the path moves down, and a down-step every time the path moves to the right. For example, $\psi(2314657)=$ uuduudududddud.

The bijection $\phi$ from 132-avoiding permutations to $\mathcal{D}_{n}$ is the standard bijection followed by a reflection of the path with respect to a vertical line through the middle of the path. For example, $\phi(7432516)=$ uuduudududddud. 


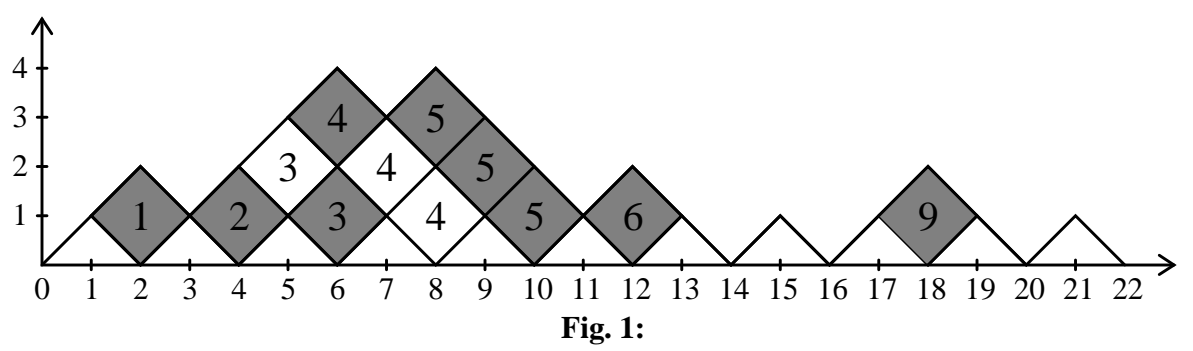

The Elizalde-Deutsch bijection is defined by Elizalde-Deutsch $=\phi^{-1} \circ \Psi^{-1} \circ \psi$. As an example, it send 2314657 to 2314657.

\subsection{Mansour-Deng-Du's bijection, 2006}

Let $i$ be a positive integer smaller than $n$. Let $s_{i}: \mathcal{S}_{n} \rightarrow \mathcal{S}_{n}$ act on permutations by interchanging the elements in positions $i$ and $i+1$. We call $s_{i}$ a simple transposition, and write the action of $s_{i}$ as $\pi s_{i}$. So, $\pi\left(s_{i} s_{j}\right)=\left(\pi s_{i}\right) s_{j}$. For any permutation $\pi$ of length $n$, the canonical reduced decomposition of $\pi$ is

$$
\pi=(12 \ldots n) \sigma=(12 \ldots n) \sigma_{1} \sigma_{2} \ldots \sigma_{k}
$$

where $\sigma_{i}=s_{h_{i}} s_{h_{i}-1} \ldots s_{t_{i}}, h_{i} \geq t_{i}, 1 \leq i \leq k$ and $1 \leq h_{1}<h_{2}<\cdots<h_{k} \leq n-1$. For example, $415263=\left(s_{3} s_{2} s_{1}\right)\left(s_{4} s_{3}\right)\left(s_{5}\right)$.

Mansour, Deng and Du (9) use canonical reduced decompositions to construct a bijection between $\mathcal{S}_{n}(321)$ and $\mathcal{S}_{n}(231)$. They show that a permutation is 321 -avoiding precicely when $t_{i} \geq t_{i-1}+1$ for $2 \leq i \leq k$ (9, Thm. 2). They also show that a permutation is 231 -avoiding precicely when $t_{i} \geq t_{i-1}$ or $t_{i} \geq h_{i-j}+2$ for $2 \leq i \leq k$ and $1 \leq j \leq i-1$ (9, Thm 15). Using these two theorems they build their bijection, which is composed of two bijections: one from $\mathcal{S}_{n}(321)$ to $\mathcal{D}_{n}$, and one from $\mathcal{S}_{n}(231)$ to $\mathcal{D}_{n}$.

For a Dyck path $P$, we define the $(x+y)$-labelling of $P$ as follows: each cell in the region enclosed by $P$ and the $x$-axis, whose corner points are $(i, j),(i+1, j-1),(i+2, j)$ and $(i+1, j+1)$ is labelled by $(i+j) / 2$. If $(i-1, j-1)$ and $(i, j)$ are starting points of two consecutive up-steps, then we call the cell with leftmost corner $(i, j)$ an essential cell and the up-step $((i-1, j-1),(i, j))$ its left arm. We define the zigzag strip of $P$ as follows: If there is no essential cell in $P$, then the zigzag strip is simply the empty set. Otherwise, we define the zigzag strip of $P$ as the border strip that begins at the rightmost essential cell. For example, the zigzag strip of the Dyck path uuduuududddudduduuddud in Figure 1 is the shaded cell labelled by 9, while for the Dyck path uuduuududdd (obtained from that in Figure 1 by ignoring the steps 15 to 22) the zigzag strip is the shaded connected cells labelled by $2,3,4,5$ and 6 .

Let $P_{n, k}$ be a Dyck path of semilength $n$ containing $k$ essential cells. We define its zigzag decomposition as follows: The zigzag decomposition of $P_{n, 0}$ is the empty set. The zigzag decomposition of $P_{n, 1}$ is the zigzag strip. If $k \geq 2$, then we decompose $P_{n, k}=P_{n, k-1} Q$, where $Q$ is the zigzag strip of $P_{n, k}$ and $P_{n, k-1}$ is the Dyck path obtained from $P$ by deleting $Q$. Reading the labels of $Q$ from left to right, ignoring repetitions, we get a sequence of numbers $\{i, i+1, \ldots, j\}$, and we associate $Q$ with the sequence of simple decompositions $\sigma_{k}=s_{j} s_{j-1} \ldots s_{i}$. For $P_{n, i}$ with $i \leq k-1$ repeat the above procedure to get $\sigma_{k-1}, \ldots, \sigma_{2}, \sigma_{1}$. The zigzag decomposition of $P_{n, k}$ is then given by $\sigma=\sigma_{1} \sigma_{2} \ldots \sigma_{k}$.

From the zigzag decomposition we get a 321 -avoiding permutation $\pi=(12 \ldots n) \sigma$ whose canonical 


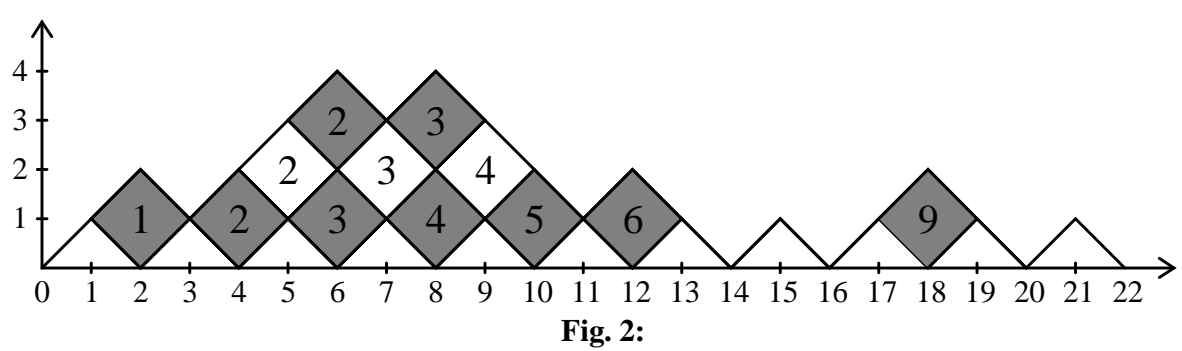

reduced decomposition is $\sigma$. For the Dyck path $P_{11,4}$ in Figure 1 we have

$$
\sigma=\left(s_{3} s_{2} s_{1}\right)\left(s_{4} s_{3}\right)\left(s_{6} s_{5} s_{4}\right)\left(s_{9}\right)
$$

and the corresponding permutation in $\mathcal{S}_{11}(321)$ is $(4,1,5,7,2,3,6,8,10,9,11)$.

We will now describe a map from Dyck paths to 231 -avoiding permutations. For a Dyck path $P$, we define $(x-y)$-labelling of $P$ as follows (this labelling seems to be considered for the first time in (1)): each cell in the region enclosed by $P$ and the $x$-axis, whose corner points are $(i, j),(i+1, j-1),(i+2, j)$ and $(i+1, j+1)$ is labelled by $(i-j+2) / 2$. We define the trapezoidal strip of $P$ as follows: If there is no essential cell in $P$, then the trapezoidal strip is simply the empty set. Otherwise, we define the trapezoidal strip of $P$ as the horizontal strip that touches the $x$-axis and starts at the rightmost essential cell. For example, the trapezoidal strip of the Dyck path uuduuududddudduduuddud in Figure 2 is the shaded cell labelled by 9 , while for the Dyck path uuduuududdd (obtained from that in Figure 2 by ignoring the steps 15 to 22 ) the zigzag strip is the downmost shaded strip with labels $1,2,3,4,5$ and 6 .

Let $P_{n, k}$ be a Dyck path of semilength $n$ containing $k$ essential cells. We define its trapezoidal decomposition as follows: The trapezoidal decomposition of $P_{n, 0}$ is the empty set. The trapezoidal decomposition of $P_{n, 1}$ is the trapezoidal strip. If $k \geq 2$, then we decompose $P_{n, k}$ into $P_{n, k}=Q_{1} u Q_{2} d$, where $u$ is the left arm of the rightmost essential cell that touches the $x$-axis, $d$ is the last down step of $P_{n, k}$, and $Q_{1}$ and $Q_{2}$ carry the labels in $P_{n, k}$. Reading the labels of the trapezoidal strip of $P_{n, k}$ from left to right we get a sequence $\{i, i+1, \ldots, j\}$, and we set $\sigma_{k}=s_{j} s_{j-1} \ldots s_{i}$. Repeat the above procedure for $Q_{1}$ and $Q_{2}$. Suppose the trapezoidal decomposition of $Q_{1}$ and $Q_{2}$ are $\sigma^{\prime}$ and $\sigma^{\prime \prime}$ respectively, then the trapezoidal decomposition for $P_{n, k}$ is $\sigma=\sigma^{\prime} \sigma^{\prime \prime} \sigma_{k}$.

From the trapezoidal decomposition we get a 231-avoiding permutation $\pi=(12 \ldots n) \sigma$ whose canonical reduced decomposition is $\sigma$. For the Dyck path $P_{11,4}$ in Figure 2 we have

$$
\sigma=\left(s_{3} s_{2}\right)\left(s_{4} s_{3} s_{2}\right)\left(s_{6} s_{5} s_{4} s_{3} s_{2} s_{1}\right)\left(s_{9}\right)
$$

and the corresponding permutation in $\mathcal{S}_{11}(231)$ is $(7,1,5,4,2,3,6,8,10,9,11)$.

The two maps involving Dyck paths described in this subsection induce a bijection from 321-avoiding to 231-avoiding permutations.

\section{A recursive description of Knuth-Richards' bijection}

We call a permutation $\pi$ indecomposable if $\operatorname{comp}(\pi)=1$; otherwise we call $\pi$ decomposable. Equivalently, if we define the sum $\oplus$ on permutations by $\sigma \oplus \tau=\sigma \tau^{\prime}$, where $\tau^{\prime}$ is obtained from $\tau$ by adding 
$|\sigma|$ to each of its letters, then a permutation is indecomposable if it cannot be written as the sum of two nonempty permutations.

We shall describe, separately for 231- and 321-avoiding permutations, how to generate the indecomposable permutations, thus inducing a bijection we call $\Phi$.

For a permutation of length $n$ to be 231-avoiding everything to the left of $n$ has to be smaller than anything to the right of $n$. Clearly, if there is at least one element to the left of $n$, then the permutation is decomposable (everything to the right of $n$, including $n$, would form the last component). Thus a 231 -avoiding permutation of length $n$ is indecomposable if and only if it starts with $n$.

To build an indecomposable 231-avoiding permutation of length $n$ from a 231-avoiding permutation of length $n-1$ we simply prepend $n$. Let us call this map $\alpha$. For instance, $\alpha(2134)=52134$.

Given $k$ indecomposable 231 -avoiding permutations $\pi_{1}, \pi_{2}, \ldots, \pi_{k}$, we build the corresponding permutation by summing: $\pi_{1} \oplus \pi_{2} \oplus \cdots \oplus \pi_{k}$. Given $k$ indecomposable 321 -avoiding permutations $\pi_{1}, \pi_{2}, \ldots, \pi_{k}$ we build the corresponding permutation by summing in reverse order: $\pi_{k} \oplus \pi_{k-1} \oplus \cdots \oplus \pi_{1}$.

Here is how we build an indecomposable 321-avoiding permutation $\pi^{\prime}$ of length $n$ from a 321-avoiding permutation $\pi$ of length $n-1$ :

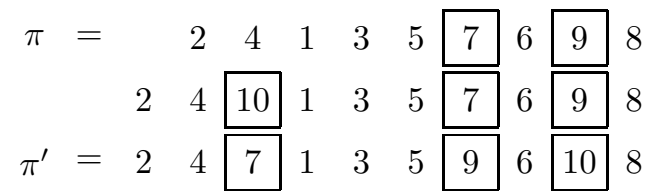

In the first row we box the left-to-right maxima to the right of 1 that are not right-to-left minima. Here, those are 7 and 9 . In the second row we insert a new largest element, 10, immediately to the left of 1 and box it. Finally, in the third row, we cyclically shift the sequence of boxed element one step to the left, thus obtaining $\pi^{\prime}$. Let us call this map $\beta$.

The induced map $\Phi$, between 231 - and 321-avoiding permutations is then formally defined by

$$
\Phi(\epsilon)=\epsilon ; \quad \Phi(\alpha(\sigma))=\beta(\Phi(\sigma)) ; \quad \Phi(\sigma \oplus \tau)=\Phi(\tau) \oplus \Phi(\sigma) .
$$

As an example, consider the permutation 5213476 in $\mathcal{S}_{6}(231)$. Decompose it using $\oplus$ and $\alpha$ :

$$
5213476=52134 \oplus 21=\alpha(2134) \oplus \alpha(1)=\alpha(\alpha(1) \oplus 1 \oplus 1) \oplus \alpha(1) .
$$

Reverse the order of summands and change each $\alpha$ to $\beta$ :

$$
\beta(1) \oplus \beta(1 \oplus 1 \oplus \beta(1))=21 \oplus \beta(1243)=21 \oplus 41253=2163475 .
$$

In conclusion, $\Phi(5213476)=2163475$.

\section{References}

[1] J. Bandlow and K. Killpatrick, An area-to-inv bijection between Dyck paths and 312-avoiding permutations, Elect. J. Combin. 8 (2001) \#R40.

[2] A. Claesson, S. Kitaev and E. Steingrímsson, Stack sorting, trees, and pattern avoidance, arXiv:0801.4037v1 [math.CO]. 
[3] I. P. Goulden and J. West, Raney paths and a combinatorial relationship between rooted nonseparable planar maps and two-stack-sortable permutations, J. Combin. Theory Ser. A 75 (1996), 200-242.

[4] S. Elizalde and E. Deutsch, A Simple and Unusual Bijection for Dyck Paths and its Consequences, Annals of Comb. 7 (2003), 281-297.

[5] S. Elizalde and I. Pak, Bijections for refined restricted permutations, J. Comb. Theory, Ser. A 105(2) (2004), 207-219.

[6] D. E. Knuth, The art of computer programming, Vol. I Fundamental algorithms. Addison-Wesley Publishing Co., Reading, Mass.-London-Don Mills, Ont, 1969.

[7] D. E. Knuth, The art of computer programming, Vol. III, Addison-Wesley, Reading, MA, 1973.

[8] C. Krattenthaler, Permutations with restricted patterns and Dyck paths, Adv. Appl. Math. 27 (2001), $510-530$.

[9] T. Mansour, E. Deng and R. Du, Dyck paths and restricted permutations, Discrete Appl. Math. 154 (2006), 1593-1605.

[10] A. Reifegerste, A generalization of Simion-Schmidt's bijection for restricted permutations, Electron. J. Combin. 9 (2002), no. 2, Paper \#R14, 9 pp. (electronic).

[11] D. Richards, Ballot sequences and restricted permutations, Ars Combin. 25 (1988), 83-86.

[12] A. Robertson, D. Saracino and D. Zeilberger, Refined Restricted Permutations, Ann. Comb. 6 (2003), 427-444.

[13] D. Rotem, On a correspondence between binary trees and a certain type of permutations, Information processing letters 4, no. 3 (1975), 58-61.

[14] R. Simion and F. W. Schmidt, Restricted Permutations, Europ. J. Combin. 6 (1985), 383-406.

[15] J. West, Permutation trees and the Catalan and Schröder numbers Discrete Math., 146 (1995), $247-$ 262.

[16] D. Zeilberger: A proof of Julian West's conjecture that the number of two-stack-sortable permutations of length $n$ is $2(3 \mathrm{n}) ! /((\mathrm{n}+1) !(2 \mathrm{n}+1) !)$, Discrete Math. $102(1992), 85-93$. 